\title{
RELAÇÕES DE INTERAÇÃO, COMUNICAÇÃO E DESEMPENHO ENTRE MEMBROS DE EQUIPES VIRTUAIS: UMA REVISÃO BIBLIOGRÁFICA SISTEMÁTICA
}

\author{
Relationships of Interaction, Communication and Performance among members \\ of virtual teams: A Systematic Bibliographic Review \\ Gabriela Kuhnen, Célio T. dos Santos
}

Comunicação, interação, desempenho, equipes virtuais

As equipes virtuais, assim como o trabalho remoto, tornaram-se de grande importância para as empresas, pois são capazes de atender as exigências competitivas do mercado e concorrência global. Porém, a mediação da tecnologia e os novos processos comunicacionais podem afetar o desempenho da equipe. Este artigo busca revisar parte da literatura, por meio de uma revisão bibliográfica sistemática, sobre as relações da mediação tecnológica na comunicação e sua influência no desempenho de equipes, apontando quais são as principais dificuldades e aspectos na interação dos membros e o que a diferem das tradicionais. Os principais tópicos desenvolvidos pelos artigos foram em relação às tecnologias e sua influência nos processos comunicacionais, mediados pela interdependência de tarefas, assim como a virtualidade moderadora da confiança e satisfação e interação entre os membros.

\section{Communication, Performance, Virtual Teams}

Virtual teams, as well as remote work, have become of great importance to companies as they are able to meet the competitive requirements of the market and global competition. However, mediation of technology and new communication processes can affect team performance. This article seeks to review part of the literature, through a systematic literature review, on the relations of technological mediation in communication and its influence on team performance, pointing out the main difficulties and aspects in the interaction of the members and what differ them from the Traditional. The main topics developed by the articles were related to the technologies and their influence on the communication processes, mediated by the interdependence of tasks, as well as the moderating virtuality of trust and satisfaction and interaction among the members.

\section{Introdução}

Geograficamente distribuídas e dispersas no tempo, as equipes virtuais podem ser definidas como um grupo de indivíduos que utilizam as tecnologias de informação e comunicação (TICs) para se comunicar e colaborar (MALHOTRA, MAJCHRZAK, 2014; PLOTNICK, HILTZ, PRIVMAN, 2016). Unidos em prol de projetos ou objetivos específicos, trazem algumas vantagens como: equipes multidisciplinares, diminuição de certos custos (PAUL, DRAKE, LIANG, 2016), em estruturas flexíveis e menos hierarquizadas, possibilitando maior rapidez no desenvolvimento de produtos e serviços e criando maiores chances de inovação, por permitir a interação de pessoas com especialidades e expertises em áreas diversas. Porém, esse meio traz certas especificidades na interação social e relacional, já que ocorre onde a comunicação é mediada por computador e pelas tecnologias. (HAMBLEY, O'NEILL, KLINE, 2007).

Essencialmente, as equipes virtuais diferem das tradicionais por serem distanciadas no espaço e por sua forma de comunicação se basear principalmente nas TICs. De tal forma, podem também ser caracterizadas pelo nível de sua virtualidade, baseado no grau de uso de ferramentas virtuais para coordenar e executar processos de equipe. (MAYNARD, GILSON, 2014; HUNSAKER, HUNSAKER, 2008).

O compartilhamento da informação e do conhecimento ocorre através dessa mediação, podendo haver perda da presença social, do contexto e de sinais não-verbais ou paraverbais. As interações e comunicações interpessoais trazem muitas diferenças das equipes presenciais e podem influenciar o desempenho e a satisfação da equipe. Há poucas ou nenhuma interação

Anais do 8 CIDI e 8 CONGIC

Guilherme Santa Rosa; Cristina Portugal (orgs.)

Sociedade Brasileira de Design da Informação - SBDI

Natal | Brasil | 2017

ISBN 978-85-212-1305-5
Proceedings of the $8^{\text {th }}$ CIDI and $8^{\text {th }}$ CONGIC

Guilherme Santa Rosa; Cristina Portugal (orgs.)

Sociedade Brasileira de Design da Informação - SBDI

Natal| Brazil | 2017

ISBN 978-85-212-1305-5 
presencial e as mediações utilizam diferentes canais que podem permitir a transmissão síncrona ou assíncrona, trazendo aspectos específicos nessas interações. (HAMBLEY, O NEILL, KLINE, 2007; HUNSAKER, HUNSAKER, 2008).

As TICs tiveram grande impacto nas mudanças das condições de trabalho e de vida dos indivíduos, tanto nas regras e normas profissionais, como nos padrões de comunicação, nas funções e tarefas e nas estruturas organizacionais. Nesse novo sistema, é necessário maior cooperação, maior interdisciplinaridade e contatos interdepartamentais. Ao combinar todos esses elementos, tem-se uma estrutura flexibilizada e mais horizontal. Quanto maior e mais ampla for a utilização das TICs nas organizações, mais o poder se concentra em uma organização "invisível". Porém, essa nova forma de trabalho, traz impacto no ambiente psicossocial, muito mais do que em aspectos físicos. O ambiente psicossocial reflete principalmente em como os fatores objetivos do ambiente impactam na percepção individual do trabalho e de suas condições, é a interação do ambiente com o indivíduo. (HENDRICK, KLEINER, 2002).

Há muitas pesquisas e abordagens relacionadas a essas equipes, e um dos propósitos desta revisão bibliográfica sistemática é justamente entender quais são elas, a partir dos últimos dez anos, pois as tecnologias de comunicação e informação, continuam se desenvolvendo de forma significante e alterando a forma como o trabalho é conduzido. (MAYNARD, GILSON, 2014).

\section{Metodologia}

\section{Revisão bibliográfica sistemática - planejamento}

\section{Problema:}

Qual o papel da mediação tecnológica na comunicação e sua influência no desempenho de equipes?

\section{Objetivo:}

Investigar as relações da mediação tecnológica na comunicação e sua influência no desempenho de equipes, apontando quais são as principais dificuldades e aspectos na interação dos membros.

\section{Localização dos estudos:}

A principal fonte de busca utilizada foi a base de dados Scopus, segundo BURNHAM (2006), produzida pela Elsevier Co que indexam mais de 14.000 publicações de ciências sociais de 4000 editores.

\section{Execução:}

Os strings utilizados na busca foram: "virtual team" or " virtual group" or "virtual work" and communication or $\mathrm{cmc}$ and performance. O primeiro resultado revelou 318 documentos.

\section{Avaliação crítica:}

Dentre os resultados encontrados, foram selecionados apenas os artigos publicados em periódicos, resultando em 141 documentos. Excluíram-se os artigos de ciências da computação por não participarem do escopo desta pesquisa, por serem de conteúdo técnico das ciências exatas, não abrangendo muitos aspectos relacionados às ciências sociais, resultando em 95 artigos. Após essa seleção, os documentos foram filtrados a partir do título e foram excluídos os que não mencionaram nenhum tipo de relação com o objetivo da investigação, restando 65 documentos. O filtro seguinte foi determinado pela delimitação histórica: últimos 10 anos e , por se tratar de um assunto que está intimamente relacionado com mudanças das tecnologias. $\mathrm{E}$ por fim, foram selecionados os artigos de interesse pelo resumo: considerando aspectos que configurassem interesse de acordo com os objetivos e problema planejados. A pesquisa resultou então em 15 artigos. Durante o exame do conteúdo e corpo do texto, foram excluídos mais 4 artigos, por não estarem diretamente relacionados com o objeto de estudo especificado e trazerem relações mais profundas da psicologia.

Tabela1: Delimitação dos resultados encontrados. Fonte: Elaborado pelos autores. 


\section{CIDI2017 $\quad 8^{\text {th }}$ CIDI

FILTRO

STRINGS: "virtual team" or " virtual group" or "virtual work" and communication or $\mathrm{cmc}$ and performance.

ARTIGOS PUBLICADOS EM PERIÓDICOS

EXCLUÍDA ÁREA: CIÊNCIAS DA COMPUTAÇÃO

TÍTULO

DELIMITAÇÃO HISTÓRICA: ÚLTIMOS 10 ANOS E ANÁLISE DE RESUMOS

\section{ANÁLISE DE CONTEÚDO E CORPO DO TEXTO}

\section{RESULTADOS}

318 DOCUMENTOS

141 RESULTADOS

95 RESULTADOS

65 RESULTADOS

15 DOCUMENTOS

11 DOCUMENTOS

Coleta dos dados:

Dos 11 artigos selecionados, foram verificados os seguintes aspectos:

\section{Distribuição Histórica:}

Quanto à distribuição histórica, percebe-se que houve inconstâncias de publicações. Pôde-se notar que a maioria dos artigos são recentes, com o ápice nos anos de 2014 e 2016 com 5 e 4 artigos publicados respectivamente, o que indica maior relevância em pesquisas recentes e contribui significativamente para a pesquisa, conforme gráfico 1 abaixo:

Gráfico 1: Distribuição histórica dos artigos selecionados.

Fonte: Elaborado pela autora.

\section{Distribuição histórica}

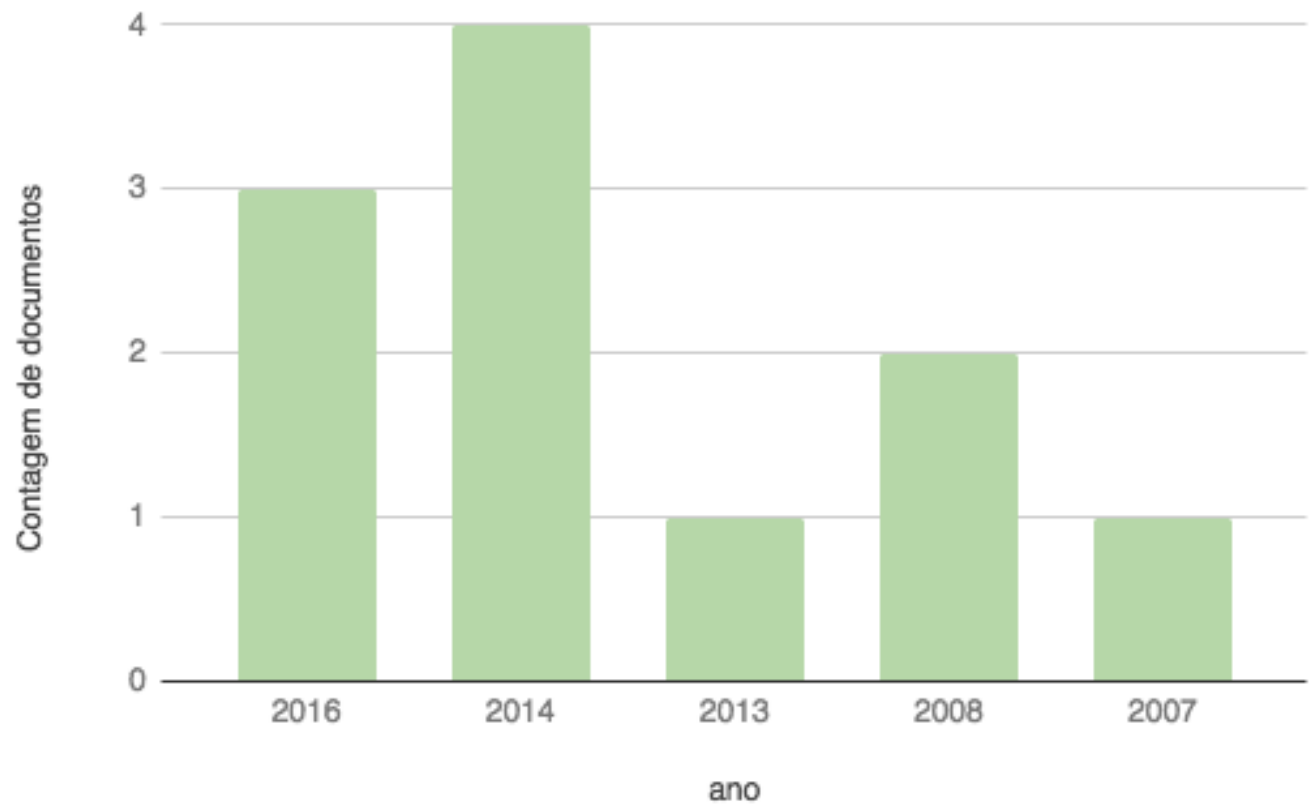




\section{Resultados}

\section{Análise e apresentação dos dados}

\section{Virtualidade e tecnologias:}

As tecnologias fornecem os canais de comunicação entre membros de equipes virtuais, nesse caso, a mediação pode ser, ao mesmo tempo, em locais diferentes (síncrona), ou em tempo diferentes e diferente localização (assíncrona). A frequência e possibilidade de se comunicar face a face são aspectos importantes na comunicação (WEIMANN, ET AL, 2013). As equipes podem ser caracterizadas também quanto a sua virtualidade, já que, o crescimento exacerbado do uso das tecnologias, para coordenar e executar projetos, predomina nas organizações modernas. Os aspectos predominantes da virtualidade são o grau de sincronização e a capacidade de transmitir aspectos não verbais e paraverbais. Quanto maior essa capacidade, menor sua virtualidade, pois mais se assemelha à comunicação presencial. (DE JONG, SCHALK, CURSEU, 2008).

As pesquisas das tecnologias na comunicação têm gerado algumas classificações: A teoria da riqueza dos meios de comunicação categoriza o canal conforme a possibilidade de transmitir informações não verbais e paraverbais, como na comunicação presencial de linguagem natural. Quanto maior essa possibilidade, mais rico é o canal. Em geral, classificase o tipo de tarefa de acordo com a riqueza. (WEIMANN, ET AL, 2013).

Outra teoria é a da Sincronicidade que categoriza os canais de acordo com os atributos de possibilitar, ou não, convergência de significados. A comunicação pode ser apenas de transporte, informar sobre algo, sem necessitar a convergência. Nessa teoria, o grau de uso deve ser separado da forma ou tipo de uso. Para cada parte da tarefa, uma tecnologia pode ser mais indicada do que outra, dependendo do intuito da comunicação, de tal forma que a efetividade depende da escolha da mídia em relação à tarefa. (HENDERSON, STACKMAN, LINDEKILDE, 2016; HUNSAKER, HUNSAKER, 2008). Resultados de Collins, Chou e Warner (2014) demonstram isso:

Tabela 4: Resultados da variável canal de comunicação Fonte: Elaborado pelos autores.

O tipo de ferramenta tecnológica
Impacto na interação de cooperação
(COLLINS, CHOU, WARNER, 2014)

\section{Efeitos da comunicação no desempenho em equipes virtuais:}

O processo comunicacional é um fator importante no desempenho de equipes virtuais, pois é, através do mesmo, que se dá o compartilhamento de informações, tanto pessoal, como da tarefa, ou do processo. Diferenças culturais e interações podem ser afetadas pela mediação tecnológica, justamente pela falta de presença social ou contextual no qual estão inseridos. Tais aspectos, são mais facilmente partilhados, na comunicação presencial. A transmissão de sinais verbais, não-verbais e paraverbais impactam em como essa informação é recebida, sua qualidade e em que tipo de interação irá cultivar. (COLLINS, CHOU, WARNER, 2014).

O desempenho de equipes virtuais é uma variável que tem sido bastante recorrente em pesquisas científicas. Muitos são os fatores que podem influenciar seus resultados de eficiência e eficácia na equipe. Alguns aspectos críticos das equipes virtuais, como a mediação da tecnologia, adaptação cultural e comunicação interpessoal, merecem certo destaque. Outros aspectos mais tangíveis também podem ser mensurados, como tempo e utilização dos recursos. (CHANG, HUNG, HSIEH, 2014; PLOTNICK, HILTZ, PRIVMAN, 2016).

O desempenho pode ser tanto do processo da equipe quanto do resultado da tarefa como, por exemplo, um produto. Refere-se à qualidade, à funcionalidade e ao impacto do produto. $O$ desempenho do processo, no entanto, pode ser visto como a coesão da equipe, sendo mensurado pela grau de intenção dos membros de permanecer trabalhando juntos, percepção compartilhada de propósitos, como normas, valores e objetivos e a satisfação dos membros da equipe. Outros processos são mencionados no desempenho, como os de interação, também relacionados à motivação e aos conflitos entre os membros. Todas as pesquisas mencionadas 
corroboram, de alguma forma, que a tecnologia é um dos fatores mediadores do desempenho. (HAMBLEY, O’NEILL, KLINE, 2007); (PLOTNICK, HILTZ, PRIVMAN, 2016); (PAUL, DRAKE, LIANG, 2016).

\section{Aspectos psicossociais: \\ Confiança:}

Está relacionada à vulnerabilidade das ações em relação às outras partes, suas crenças e expectativas em relação aos outros, que todos ajam em conformidade às regras e normas préestabelecidas e ao trabalho colaborativo, para obter os resultados em comum. A confiança entre membros de equipes virtuais podem diminuir conflitos, influenciando nos resultados e desempenho da equipe. Alguns dos aspectos relacionados à confiança são: interações face a face, que forneçam um grau mais elevado de presença social, normas e regras bem estabelecidas, como a clareza das atribuições de cada membro e objetivos do projeto, respostas oportunas, comunicação aberta, feedbacks, interações previsíveis e consistentes. Estes aspectos estão relacionados positivamente com o nível de confiança (CHANG, HUNG, HSIEH, 2014); (PLOTNICK, HILTZ, PRIVMAN, 2016; PAUL, DRAKE, LIANG, 2016; HENDERSON, STACKMAN, LINDEKILDE, 2016; HUNSAKER, HUNSAKER, 2008), e alguns resultados são demonstrados na tabela abaixo:

Tabela 5: Resultados relacionados à variável confiança Fonte: Elaborado pelos autores.

\begin{tabular}{|l|l|l|}
\hline Maior confiança & Maior desempenho da equipe & (PAUL, DRAKE, LIANG, 2016) \\
\hline Maior confiança & Maior coesão da equipe & (PAUL, DRAKE, LIANG, 2016)
\end{tabular}

\section{Diferenças culturais:}

Membros de equipes virtuais alocados em regiões geográficas distintas, de forma, principalmente global, como diferentes nacionalidades, trazem aspectos culturais bastante divergentes: maneiras de interagir, de se relacionar e de se comunicar. Nesse caso, a convergência de normas e significados pode apresentar mais dificuldades de adesão, e tais aspectos podem fazer com que os membros difiram em expectativas ou escolhas das mídias. A utilização das TICs pode amplificar a diversidade cultural nos contextos heterogêneos de equipes distanciadas geograficamente, criando maiores desentendimentos e conflitos, pois podem estar presentes nas habilidades de comunicação, éticas de trabalho e maneiras de resolver problemas. (HENDERSON, STACKMAN, LINDEKILDE, 2016; HUNSAKER, HUNSAKER, 2008; CHANG, HUNG, HSIEH, 2014). Através de alguns ajustes, aprendizagem, colaboração e processo de compreensão, é possível estabelecer um alto nível de adaptação cultural, conforme resultados da pesquisa de Chan, Hung, Hsieh (2014), descritos na tabela abaixo. Esta adaptação é importante para que se possa mediar conflitos e desconfianças, fazendo crescer o desempenho da equipe.

Tabela 6: Resultados referentes à variável adaptação cultural Fonte: Elaborado pelos autores.

\begin{tabular}{|l|l|l|}
\hline Maior adaptação cultural & $\begin{array}{l}\text { Maior a qualidade da } \\
\text { comunicação }\end{array}$ & (CHANG, HUNG, HSIEH, 2014) \\
\hline Maior adaptação cultural & Maior confiança & (CHANG, HUNG, HSIEH, 2014) \\
\hline Maior adaptação cultural & $\begin{array}{l}\text { Maior desempenho da } \\
\text { equipe }\end{array}$ & (CHANG, HUNG, HSIEH, 2014) \\
\hline
\end{tabular}

Satisfação: 
A satisfação dos indivíduos, está relacionada com a possibilidade de a equipe prover satisfação aos membros, através de oportunidades de crescimento e desenvolvimento pessoal. Muitos são os fatores que podem influenciar nessa variável, como normas, funções bem estabelecidas ou clareza na comunicação. (WEIMANN, ET AL, 2013; HENDERSON, STACKMAN, LINDEKILDE, 2016). Em geral, essa é a variável dependente que irá modificar-se de acordo com a influência de variáveis independentes ou moderadoras.

\section{Coesão:}

Está relacionada à interação e à integração entre os membros, com a percepção conjunta das normas, valores e objetivos. (PAUL, DRAKE, LIANG, 2016).

Nos estudos de Plotinick, Hiltz e Privman (2016), a dinâmica do grupo é tratada como um processo que ocorre dentro de subgrupos que trabalhem de forma presencial com outros subgrupos que interagem de forma virtual, fazendo com que se perca parte do sentido da equipe como um todo. Pode ocorrer que membros da equipe exibam uma atitude favorável a seu subgrupo e gerem estereótipos negativos dos demais, produzindo conflitos e gerando a falta de uma identidade e de modelos mentais compartilhados. A dinâmica do grupo foi medida através das variáveis conflito, confiança e integridade reduzida entre os subgrupos.

Corroborada pela pesquisa de Paul, Drake e Liang (2016) que mede a coesão da equipe, considerando aspectos como interação entre os membros, percepção conjunta de normas, valores e os objetivos em relação ao desempenho geral. Percebe-se, assim, que a falta de identidade social, que pode ser gerada pela comunicação mediada pelas tecnologias, diminui a eficácia percebida da equipe. Em relação a isso, a utilização das tecnologias com qualidade e confiança pode aumentar a identidade percebida. E quanto mais cooperativos são os processos de interação entre os membros, maior é a confiança e o desempenho da equipe. Os resultados das pesquisas são demonstrados na tabela abaixo: Tabela 7: Resultados encontrados em relação à variável coesão e processos da equipe
Fonte: Elaborado pelos autores.
Menor a falta de identidade social da equipe
Maior eficácia percebida
(PLOTNICK, HILTZ, PRIVMAN, 2016)
Maior o treinamento e instrução da equipe
Menor será a falta de
(PLOTNICK, HILTZ, PRIVMAN, identidade percebida 2016)
A confiabilidade e suporte à
Aumenta a identidade tecnologia de comunicação percebida
(PLOTNICK, HILTZ, PRIVMAN, 2016)
Maior coesão
Maior confiança
(PAUL, DRAKE, LIANG, 2016)
Maior coesão global da equipe
Maior o desempenho
(PAUL, DRAKE, LIANG, 2016)

No do processo de coesão da equipe, podem-se destacar outras variáveis que são investigadas de forma mais isoladas, a saber:

\section{Interações em equipe:}

As interações referem-se aos processos construídos entre os interagentes e levam ao engajamento e socialização dos envolvidos. São entendidas, também, através dos padrões de comunicação utilizados para solucionar conflitos de tarefas e manter as relações entre os membros. Podem ser classificadas como cooperativas ou competitivas. Na cooperativa, as normas, objetivos e valores são compartilhados e geram experiências positivas. Os resultados pessoais e de equipe estão em consonância, há a livre troca de informações, criatividade e respeito pela percepção dos demais membros. Na competitiva, os valores, normas e metas são divergentes, e as experiências tendem a ser negativas. São subclassificadas como passivas ou agressivas. As passivas incluem limitação do compartilhamento da informação, falta de criatividade, questionamentos, imparcialidade e harmonia. As agressivas têm ênfase 
em um comportamento voltado para os objetivos e ambições pessoais num cenário de críticas, interrupções e impaciência com outras perspectivas. Estudos demonstram certas relações com as interações e as tecnologias. Interações face a face, que produzem relações mais construtivas, elevam o desempenho da equipe, e interações mais passivas face a face, ao contrário, diminuem o desempenho; já, interações agressivas mediadas pela tecnologia tendem a produzir menos prejuízos que as presenciais (PAUL, DRAKE, LIANG, 2016; HAMBLEY, O'NEILL, KLINE, 2007). Estudos de Hambley, O'Neill, Kline (2007) procurou estabelecer relações entre os estilos de interação e as mídias utilizadas e chegou aos resultados da tabela abaixo:

Tabela 8:Resultados da variável interação Fonte: Elaborado pelos autores.

A comunicação presencial (mídia mais rica) resulta em um estilo de interação construtiva mais do que (HAMBLEY, O'NEILL, KLINE, 2007) através de videoconferências.

A comunicação presencial (mídia mais rica) resulta em um estilo de interação construtiva maior do que através de chats.

(HAMBLEY, O’NEILL, KLINE, 2007)

Falta de comunicação entre os membros afeta negativamente a interação cooperativa.

(COLLINS, CHOU, WARNER, 2014)

Interação pobre entre os membros diminui a satisfação da equipe.

(COLLINS, CHOU, WARNER, 2014)

\section{Conflitos:}

Os conflitos podem ser definidos como dissociação de percepções, discrepâncias, divergência de significados, interesses e objetivos. No trabalho em equipe, podem ser classificados de três formas: o conflito de relacionamento (interpessoal - percepções diferentes); conflito de tarefa (desentendimentos no que se refere à tarefa); conflito de processo (métodos e procedimentos adotados no funcionamento da equipe para realização dos objetivos)., (DE JONG, SCHALK, CURSEU, 2008). Conflitos de tarefa podem gerar impacto positivo no desempenho percebido da equipe, pois fazem com que as discussões ou dissociações resultem em mais pesquisas e alternativas na resolução da tarefa ou objetivo, o que ocorre, mais frequentemente, em equipes com elevado grau de virtualidade, conforme resultados da tabela abaixo:

Tabela 9: Resultados em relação a variável conflito Fonte: Elaborado pelos autores.

Os conflitos de tarefa que acontecem com equipes com elevado grau de virtualidade.

Os conflitos de tarefa que acontecem com equipes com baixo grau de virtualidade.
Impacto positivo no desempenho percebido da equipe.

Impacto negativo no desempenho percebido da equipe.
(DE JONG, SCHALK, CURSEU, 2008)

(DE JONG, SCHALK, CURSEU, 2008)

\section{Conflitos de processo}

Impacto negativo no desempenho percebido da equipe.
(DE JONG, SCHALK, CURSEU, 2008) 
A comunicação presencial ou face a face
Diminuem os conflitos e desentendimentos de equipes com diferenças culturais.
(COLLINS, CHOU, WARNER, 2014)

\section{Normas, coordenação e instrução:}

O ambiente e as funções do projeto são essenciais no desempenho da equipe. $O$ estabelecimento de normas no início do projeto, assim como o alinhamento das tarefas com as tecnologias de acordo com o contexto de cada equipe, com o estágio de cada projeto ou tarefa e com a experiência e aprendizagem dos membros podem ter influências importantes nos resultados (WEIMANN, ET AL, 2013). A coordenação se estabelece pelo grau de esforço de se manter o compartilhamento de informações e sincronização de atividades de forma consistente e lógica. Nas equipes virtuais, esses esforços de comunicação podem se tornar mais complexos devido às fronteiras espaciais e temporais. Em equipes virtuais, as normas incluem expectativas ou códigos compartilhados sobre interações e ações, como compartilhar informações, por qual canal e com que níveis de prioridade, combinando e separando trabalhos mais colaborativos ou mais independentes.

As normas podem levar certo tempo para surgir e se efetivar de modo mais natural, ou de forma alinhada com os membros do grupo, facilitando o entendimento compartilhado. A falta de normas ou regras claras do funcionamento da equipe em projetos virtuais pode criar barreiras para uma efetiva comunicação. Quanto maior a complexidade da tarefa ou mais abstratas, a falta de regras e normas podem gerar ambiguidade, ou trazer desconfianças. Acordos e instruções sobre a escolha da mídia, para se comunicar e compartilhar conteúdos, devem estar claros e facilitam o entendimento mútuo, possibilitando os modelos mentais compartilhados. (HENDERSON, STACKMAN, LINDEKILDE, 2016; HUNSAKER, HUNSAKER, 2008; PLOTNICK, HILTZ, PRIVMAN, 2016; PAUL, DRAKE, LIANG, 2016).

Quando as normas são mais culturais e menos globais, maior a dificuldade de se ter uma comunicação com qualidade, bem como a satisfação de todos os membros. A união de esforços para atender os objetivos de forma lógica e coerente pode trazer grandes resultados no funcionamento geral da equipe, como demonstra resultados da pesquisa de Paul, Drake e Liang (2016):

Tabela 10: Resultados em relação à variável coordenação e normas

Fonte: Elaborado pelos autores.

Maior efetividade de coordenação

O alinhamento das normas de comunicação

O alinhamento das normas de comunicação
Aumenta a coesão.

(PAUL, DRAKE, LIANG, 2016)

Impacto positivo na clareza das funções e atribuições

(HENDERSON, STACKMAN, LINDEKILDE, 2016)

Impactam positivamente na confiança interpessoal.
(HENDERSON, STACKMAN, LINDEKILDE, 2016)

O trabalho em equipe é um sistema complexo que utiliza mecanismos de respostas em processos interativos. Os modelos de entrada e saída IPO (input/process/output) parecem não compreender todos os aspectos dessa relação. Outros modelos, como o IMOI (input/mediators/outputs/inputs), preveem que novas entradas são realizadas pelo receptor em um processo interativo de resposta, não linear ou condicional, considerando as equipes sistemas adaptativos complexos (PAUL, DRAKE, LIANG, 2016). As entradas se iniciam nos processos de comunicação, as mediações são realizadas pelas tecnologias e as respostas e novas entradas são realizadas pelos outros membros da equipe em um processo constante e simultâneo.

Comunicação e cognição compartilhada entre equipes virtuais: 
A comunicação é um dos aspectos mais pesquisados em se tratando de equipes virtuais.

Estudos recentes abordam seu efeito considerando a interdependência da tarefa e relatam que há muita divergência quanto à influência da utilização das TICs. no desempenho das equipes. (MAYNARD; GILSON, 2014; MALHOTRA; MAJCHRZAK, 2014).

Nos estudos de Maynard e Gilson (2014), a interdependência da tarefa é utilizada para entender o desenvolvimento de modelos mentais compartilhados. Esses modelos mentais referem-se aos significados compartilhados que os membros possuem, tanto para realização da tarefa (por exemplo: recursos e tempo necessário), quanto para operar a equipe (se o líder é que terá a tomada de decisão final, regras e formas de agir). Representam o conhecimento comum entre os membros, em relação às dimensões de seus ambientes internos e externos.

As tarefas mais interdependentes exigem mais interação e colaboração dos membros, pois o resultados dependem dos esforços de todos os simultaneamente, e o trabalho pode ser alterado e combinado conforme se desenvolve. Desta forma, a convergência de significados, ou cognição compartilhada, tanto da tarefa quanto da equipe, está positivamente associada ao desempenho, moderada pela interdependência de tarefas. Ao contrário, equipes que têm divergências de significados ou conflitos de relacionamento diminuem seu desempenho. (MAYNARD; GILSON, 2014; DE JONG; SCHALK; CURSEU, 2008; HUNSAKER, HUNSAKER, 2008).

Esta hipótese, de certa forma, é corroborada pela a pesquisa de Malhotra e Majchrzak (2014), uma vez que destaca serem as tarefas não rotineiras menos propensas a terem um modelo mental compartilhado em equipes virtuais, tanto pela dificuldade de comunicação, como por serem geograficamente distribuídas. Enfatiza que as TICs devem ser orientadas a proporcionar uma convergência de significado, principalmente em tarefas não rotineiras, associando, então, o uso das TICs ao desempenho da equipe, visando, justamente, à convergência de significado. Os autores ressaltam ainda que a dependência das TICs, ou o nível de virtualidade ligado ao desempenho, depende de que forma elas são utilizadas.

Porém, pesquisas defendem que o conflito de tarefas pode aumentar o desempenho da equipe, no sentido em que faz com que se obtenham soluções e inovações na busca de um consenso de todos os membros, chamada também de conflito colaborativo. Aumenta a participação e faz com que os indivíduos sejam mais ativos no processo. (DE JONG; SCHALK; CURSEU, 2008; CHANG, HUNG, HSIEH, 2014).

Os estágios para uma cognição compartilhada podem, de certa forma, serem influenciados pelo uso das TICs: a contextualização (baseada em tarefas e trabalhos anteriores ou similares); a ação, responsável pela interação entre os indivíduos, provendo informação, e a triangulação da informação de diferentes fontes e formatos. Com esses elementos, os indivíduos convergem para a deliberação e familiarização dos sentidos e significados. Como esses estágios da construção de significados entre equipes virtuais acontecem em geral mediados pelas tecnologias, é necessário considerar de que forma os atributos dos canais impactam nesse processo. Por exemplo, a velocidade de transmissão impacta na ação, pois permite que ela seja mais natural e ajuda em um compartilhamento cognitivo. No mesmo sentido, tarefas não rotineiras necessitam de certos aspectos na comunicação, como o processamento de um fluxo contínuo de novas informações, o que é facilitado pela velocidade de transmissão. (MALHOTRA; MAJCHRZAK, 2014; MAYNARD; GILSON, 2014).

Já mídias, que permitam o envio de uma grande variedade de símbolos, ajudam na triangulação de significados, aumentando o compartilhamento de modelos mentais. E mídias, que facilitam a reprocessabilidade da informação, permitem uma maior contextualização. $O$ desenvolvimento de novas ideias em tarefas não rotineiras também pode ser facilitado com o uso de certas características dos meios de comunicação que permitam revisar ideias complexas, analisar dados e rever julgamentos, para determinar as próximas etapas, características de canais que possibilitem a reprocessabilidade. (MAYNARD; GILSON, 2014; MALHOTRA; MAJCHRZAK, 2014).

Estudos que não consideram esses aspectos das TICs podem associar o uso da mesma a um baixo desempenho, consideram o grau de virtualidade a partir da possibilidade de conseguir ou não transmitir sinais não-verbais e paraverbais, ou seja a alta virtualidade está associada à baixa sincronicidade, desconsiderando as demais características que podem prover e possibilitar modelos mentais compartilhados, dependendo do estágio de significação em que se encontrem. (DE JONG; SCHALK; CURSEU, 2008). 


\section{CIDI $20178^{8 \mathrm{n}|1| \mathrm{I})}$

Sendo assim, a comunicação mediada por computador, com características específicas de virtualidade, trazem efeitos no desempenho, dependendo do tipo de tarefa, sua interdependência na convergência ou divergência de significados e criação de modelos mentais compartilhados.

5. Considerações FinaNa interlocução com o legado da literatura, percebe-se que ainda são muitas as inquietações e desafios em relação aos novos sistemas sociotécnicos criados a partir do uso das TICs. Grandes mudanças estão ocorrendo nas interações e comunicações no ambiente do trabalho. É visto que as interações mediadas por computador não podem ser reconhecidas como as presenciais, devido aos muitos aspectos gerados pela assincronicidade, como pelas características e qualidades dos meios de comunicação. Explicados pela teoria da sincronicidade e da riqueza dos meios de comunicação, os canais com seus diferentes atributos podem beneficiar ou prejudicar a convergência de significados ou a cognição compartilhada, essencial para o desempenho de qualquer equipe.

Abaixo, a figura 1 pretende ilustrar como o funcionamento das equipes virtuais foi estabelecido na literatura revisitada. Principalmente relacionados aos aspectos de coesão da equipe, que abrange os modelos mentais compartilhados, as normas, valores e objetivos e percepções individuais, como a satisfação e a confiança. Os aspectos objetivos, como o resultados final do processo, não foram avaliados. Percebe-se que muitas são as variáveis envolvidas no processo e estão totalmente inter-relacionadas. Os canais, que são as ferramentas para a comunicação, possuem atributos que impactam nos estágios da cognição compartilhada, fazendo com que cada suporte facilite, ou não, a criação da consciência e manutenção da equipe.

Figura 1: Processos de equipe mediados pelas tecnologias Fonte: Desenvolvido pelos autores.

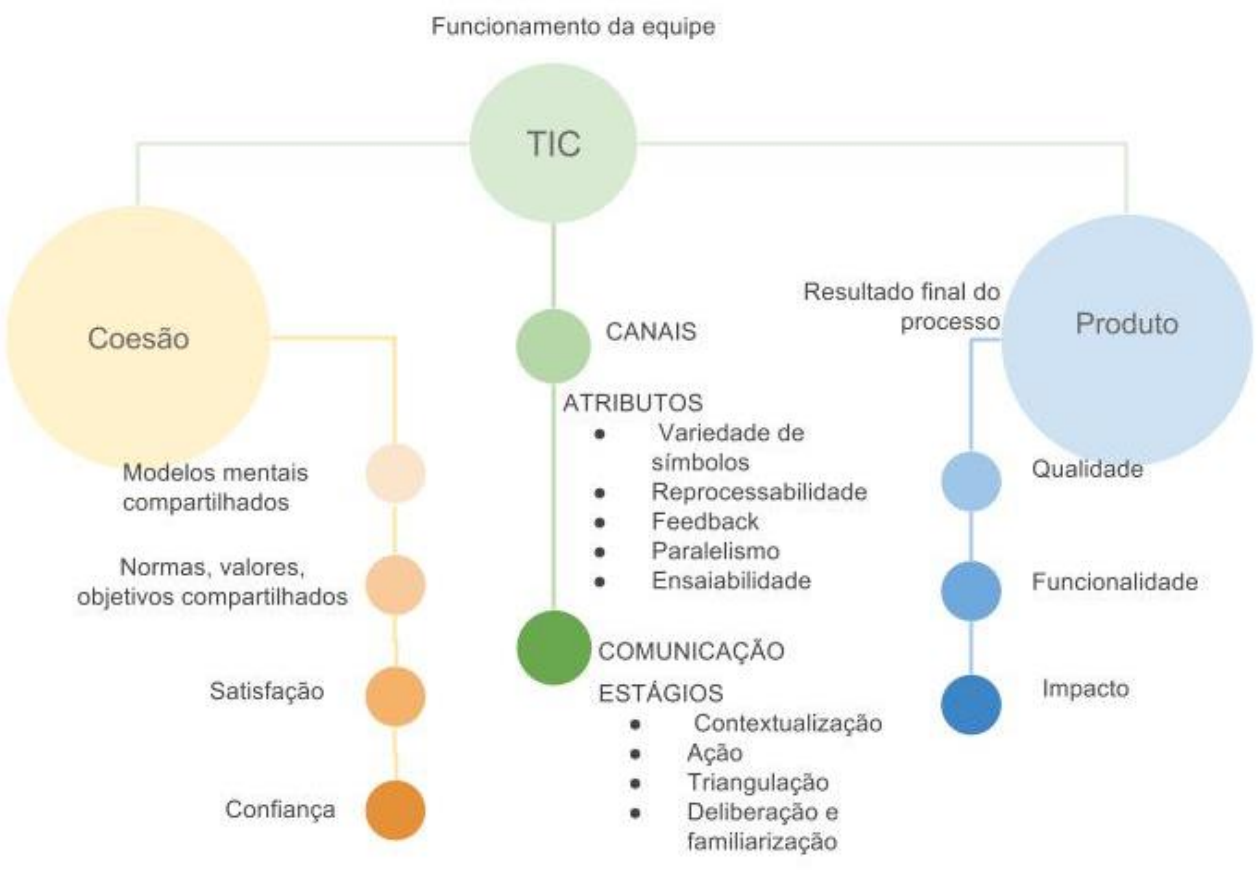

Fica claro que o desempenho das equipes pode ser medido por variáveis menos tangíveis e mais subjetivas, priorizando os aspectos psicossociais. Na literatura, percebe-se que a grande relevância é dada aos processos de desempenho relacionados à coesão da equipe, aos processos de interação entre o ambiente objetivo e a percepção individual. De fato, os trabalhos que envolvem tecnologia geram muito mais impacto no processamento das operações, das habilidades dos indivíduos, das análises e da atenção dispensada ao trabalho, do que dos aspectos físicos do ambiente.

A utilização de normas e coordenação em equipes virtuais têm o intuito de melhorar a coesão da equipe, trazendo mais satisfação, menos conflitos em relação a diferenças culturais, 
barreiras linguísticas e um compartilhamento dos objetivos. Essa coordenação também deve ser em relação aos canais de comunicação, levando em conta os aspectos mencionados referentemente à interdependência da tarefa, atributos dos meios de comunicação e criação de modelos mentais compartilhados. É necessário que a coordenação e regras bem estabelecidas façam com que todos se sintam parte da equipe, possibilitando interações mais cooperativas.

\section{Referências}

BURNHAM, Judy F. Scopus database: a review. Biomedical digital libraries, v. 3, n. 1, p. 1, 2006.

CHANG, Hsin Hsin; HUNG, Chung-Jye; HSIEH, Hsu-Wei. Virtual teams: cultural adaptation, communication quality, and interpersonal trust. Total Quality Management \& Business Excellence, v. 25, n. 11-12, p. 1318-1335, 2014.

COLLINS, Ngan; CHOU, Yu-Min; WARNER, Malcolm. Member satisfaction, communication and role of leader in virtual self-managed teamwork: case studies in Asia-Pacific region. Human Systems Management, v. 33, n. 4, p. 155-170, 2014.

CONFORTO, Edivandro Carlos; AMARAL, Daniel Capaldo; SILVA, Sérgio Luís. Roteiro para revisão bibliográfica sistemática: aplicação no desenvolvimento de produtos e gerenciamento de projetos. In: 8ํㅡㄹ Congresso Brasileiro de Gestão de Desenvolvimento de Produto-CBGDP. 2011.

DE JONG, Remco; SCHALK, René; CURŞEU, Petru L. Virtual communicating, conflicts and performance in teams. Team Performance Management: An International Journal, v. 14, n. 7/8, p. 364-380, 2008.

HAMBLEY, Laura A.; O'NEILL, Thomas A.; KLINE, Theresa JB. Virtual team leadership: The effects of leadership style and communication medium on team interaction styles and outcomes. Organizational behavior and human decision processes, v. 103, n. 1, p. 1-20, 2007.

HENDERSON, Linda S.; STACKMAN, Richard W.; LINDEKILDE, Rikke. The centrality of communication norm alignment, role clarity, and trust in global project teams. International Journal of Project Management, v. 34, n. 8, p. 1717-1730, 2016.

HENDRICK, Hal W.; KLEINER, Brian (Ed.). Macroergonomics: theory, methods, and applications. CRC Press, 2002.

HUNSAKER, Phillip L.; HUNSAKER, Johanna S. Virtual teams: a leader's guide. Team Performance Management: An International Journal, v. 14, n. 1/2, p. 86-101, 2008

MALHOTRA, Arvind; MAJCHRZAK, Ann. Enhancing performance of geographically distributed teams through targeted use of information and communication technologies. human relations, v. 67, n. 4, p. 389-411, 2014.

MAYNARD, M. Travis; GILSON, Lucy L. The role of shared mental model development in understanding virtual team effectiveness. Group \& Organization Management, v. 39, n. 1, p. 3-32, 2014.

PAUL, Ravi; DRAKE, John R.; LIANG, Huigang. Global Virtual Team Performance: The Effect of Coordination Effectiveness, Trust, and Team Cohesion. IEEE Transactions on Professional Communication, v. 59, n. 3, p. 186-202, 2016.

PLOTNICK, Linda; HILTZ, Starr Roxanne; PRIVMAN, Robin. Ingroup Dynamics and Perceived Effectiveness of Partially Distributed Teams. IEEE Transactions on Professional Communication, v. 59, n. 3, p. 203-229, 2016

WEIMANN, Peter et al. Enhancing team performance through tool use: How critical technologyrelated issues influence the performance of virtual project teams. IEEE Transactions on Professional Communication, v. 56, n. 4, p. 332-353, 2013. 
Sobre o(a/s) autor(a/es)

Nome e Sobrenome do(s) autor(ES), titulação, instituição de origem, país, e-mail.

Gabriela Kuhnen, Esp, UDESC, Florianópolis, Brasil < gabkuhnen@gmail.com>

Célio Teodorico dos Santos, Dr, Florianópolis, Brasil <celio.teodorico@gmail.com> 